\title{
GRÁFICOS ADAPTATIVOS DE CONTROLE POR ATRIBUTOS E SEU PROJETO NA PRÁTICA
}

\author{
Eugenio Kahn Epprecht * \\ Departamento de Engenharia Industrial \\ Pontifícia Univ. Católica do Rio de Janeiro (PUC-Rio) \\ eke@,ind.puc-rio.br
}

\author{
Antonio Fernando Branco Costa \\ Departamento de Produção \\ Universidade Estadual Paulista (UNESP) \\ Guaratinguetá - SP \\ Flávia Cesar Teixeira Mendes \\ Departamento de Engenharia Industrial \\ Pontifícia Univ. Católica do Rio de Janeiro (PUC-Rio) \\ * Corresponding author/autor para quem as correspondências devem ser encaminhadas \\ Recebido em 06/2004; aceito em 02/2005 após 1 revisão \\ Received June 2004; accepted February 2005 after one revision
}

\begin{abstract}
Resumo
Os gráficos de controle de processo com parâmetros variáveis, ou gráficos adaptativos, permitem intensificar ou relaxar o controle do processo de acordo com a informação da amostra mais recente. Sua vantagem é uma maior rapidez na detecção de causas especiais que tiram o processo de sua situação de controle. Este artigo apresenta um modelo geral para gráficos de $c, n p, u$ e $p$ com um, dois ou todos os parâmetros de projeto (tamanho de amostra, intervalo de tempo entre amostras e largura dos limites de controle) variáveis, que proporciona ganhos de eficiência substanciais. É proposto um procedimento para escolha dos valores para os parâmetros dos gráficos na prática, apoiado em um programa em Excel (disponível a pedido) que permite selecionar, de forma rápida e simples, dentre valores práticos, aqueles que otimizam a eficiência do gráfico de controle na detecção de alterações do processo.
\end{abstract}

Palavras-chave: gráficos de controle com parâmetros variáveis; atributos; controle estatístico de processo.

\begin{abstract}
Process control charts with variable parameters, also known as adaptive charts, allow one to intensify or to relax the control of the process according to the information about the process obtained from the most recent sample. In this paper, we study an adaptive scheme for the $c, n p, u$, and $p$ charts that allows one to vary one, two, or all design parameters (sample size, sampling interval and control limit width). The adaptive scheme increases significantly the speed with which the control chart detects process disturbances. We propose, in addition, a simple and fast procedure using an Excel spreadsheet for selection of the best values for the design parameters, from a set of practicable values.
\end{abstract}

Keywords: variable parameter control charts; attributes; statistical process control. 


\section{Introdução}

As propriedades dos gráficos de controle com parâmetros variáveis têm sido objeto de estudo de vários pesquisadores (ver Tagaras, 1998; e, em português, Costa, 1998a). Tais gráficos são chamados de gráficos de parâmetros variáveis (e, também, de gráficos adaptativos) pois um ou mais parâmetros de projeto (tamanho de amostra, intervalo de tempo entre amostras e largura dos limites de controle) variam entre dois ou mais valores, de acordo com as informações que se tem do processo. Sem aumentar o volume de inspeção, ou mesmo a freqüência de alarmes falsos, os gráficos adaptativos são ágeis na detecção de causas especiais que aumentam, de forma moderada, a incidência de nãoconformidades ou de unidades não conformes. $O$ ganho marginal de desempenho ao variar cada parâmetro de projeto do gráfico de controle entre mais de dois valores é desprezível em relação a quando eles assumem apenas dois valores (ver Reynolds et al., 1988; Reynolds, 1989, 1995; Runger \& Pignatiello, 1991; Prabhu et al., 1994; Porteus \& Angelus, 1997; e Zimmer et al., 1998); conseqüentemente, os esquemas duais têm sido os mais usuais. Nos esquemas duais, além dos limites de controle (também chamados limites de ação), o gráfico contém limites de advertência, mais estreitos. O resultado da análise de cada amostra é um valor de uma estatística particular, escolhida em função da característica do processo que se deseja monitorar. Se o resultado da análise de uma amostra gerar suspeitas de que o processo está fora de controle (um valor amostral entre os limites de advertência e de ação - ver Figura 1), o monitoramento deve ser intensificado, esperando-se assim um tempo menor do que o usual para a retirada da próxima amostra, que pode ainda ter um tamanho maior do que o usual, e cujo valor também pode ser confrontado com limites de controle mais estreitos que o usual, portanto mais sensíveis a alterações do processo. Por outro lado, se não houver suspeitas de que o processo está fora de controle (um valor amostral abaixo do limite de advertência) o monitoramento deve ser relaxado, esperando-se assim um tempo maior do que o usual para a retirada da próxima amostra, que ainda pode ter um tamanho menor do que o usual, e também pode ter seu valor confrontado com limites de controle mais largos que o usual, portanto mais robustos contra alarmes falsos. Assim, os períodos de monitoramento intenso serão compensados pelos períodos em que o monitoramento é realizado de forma branda, de tal sorte que a freqüência média de alarmes falsos e o número médio de itens inspecionados por unidade de tempo permaneçam iguais aos de um gráfico de controle tradicional, ou seja, de parâmetros fixos $(F p)$. Os gráficos adaptativos são mais ágeis no diagnóstico de causas especiais que afetam o processo de forma moderada.

Os esquemas adaptativos foram inicialmente utilizados com os gráficos de controle por variáveis. Reynolds et al. (1988) estudaram o gráfico de $\bar{X}$ com intervalo de tempo entre amostras variável (VSI - Variable Sampling Interval). O próximo parâmetro a ser considerado variável foi o tamanho de amostra: Prabhu et al. (1993) e Costa (1994) estudaram este esquema (VSS - Variable Sample Size). A direção natural de prosseguimento da pesquisa era fazer variáveis tanto o tamanho de amostra quanto o intervalo de tempo entre amostras (VSSI - Variable Sample Size and Sampling Interval), ou então fazer variáveis todos os parâmetros, incluindo o fator de abertura dos limites de controle $(\mathrm{Vp}-$ Variable Parameters). Prabhu et al. (1994) e Costa (1997, 1998a, 1999a) fornecem uma descrição detalhada destes gráficos de $\bar{X}$ com todos os parâmetros adaptativos. A idéia de usar gráficos de parâmetros variáveis foi naturalmente estendida a outros esquemas estatísticos, tais como os gráficos de $\bar{X}$ e $R$, gráficos CUSUM e EWMA (ver Reynolds et al., 1990; Saccucci et al., 1992; e Costa, 1998b, 1999b). 
A proposta de uso de esquemas adaptativos para gráficos de controle de $c, n p, u$ e $p$ é recente (ver Epprecht \& Costa, 2001; e Epprecht et al., 2003). Aqui, prefere-se o termo "gráficos adaptativos" (ou ainda "esquemas adaptativos"), a fim de distingui-los dos gráficos de $p$ ou de $u$ com parâmetros variáveis já existentes na literatura, nos quais o tamanho das amostras varia em função da disponibilidade de itens para inspeção (Montgomery (2001), p. 298-303 e 319-322; Costa et al. (2004), p. 215-217 e 225-226).

Rendtel (1990) estudou o gráfico CUSUM adaptativo por atributos, porém ele avaliou e comparou os esquemas VSS, VSI e VSSI segundo critérios diferentes dos usuais, tornando impraticável a comparação entre seu trabalho e trabalhos afins. Vaughan (1993) e Porteus \& Angelus (1997) propuseram esquemas bayesianos para controle por atributos; no entanto, os princípios, critérios e operação destes esquemas também são muito diferentes dos gráficos não-bayesianos para permitir qualquer comparação.

Este artigo apresenta o modelo de Epprecht et al. (2003) para gráficos de Shewhart adaptativos para atributos (gráficos de $c, n p, p$ e $u$ ) em que um, dois ou todos os parâmetros do gráfico variam entre dois valores. Além dos esquemas $V S S, V S I$ e $V p$, são considerados os esquemas VL ("Variable Limits"), em que só os limites de controle variam, e VSIL, em que variam o intervalo de tempo entre amostras e os limites de controle. (Vale notar que, no caso de gráficos de Shewhart para atributos, quando o tamanho de amostra varia não se consegue manter fixo o risco de alarme falso, de modo que não existe a distinção entre gráficos VSSI e $V p$ ). Para a determinação dos parâmetros destes gráficos, foi escrito um programa em Excel. Dentre valores práticos, o programa permite ao usuário selecionar, de forma rápida e simples, aqueles que maximizam a eficiência do gráfico de controle na deteç̧ão de alterações do processo.

A questão de escolha dos parâmetros (projeto) do gráfico adaptativo, fundamental para seu uso na prática, não é tratada na literatura (com raras exceções - ver, por exemplo, De Magalhães et al., 2001, 2002; e De Magalhães \& Moura Neto, 2004). Em geral, são fornecidas tabelas de projetos que apresentam bom desempenho (ou mesmo desempenho ótimo segundo algum critério), mas que, em função de restrições operacionais, podem não ser aplicáveis.

A Seção 2 descreve a operação do esquema adaptativo para o gráfico de $c$; a Seção 3 apresenta as medidas de desempenho deste gráfico, que são usadas na Seção 4 para comparação de desempenho entre o esquema proposto e o gráfico de $c$ de parâmetros fixos. A Seção 5 discute o problema do projeto do gráfico na prática, apresenta o procedimento para solução desse problema usando o programa em Excel desenvolvido pelos autores, e ilustra-o com um exemplo. A Seção 6 mostra como aplicar o esquema adaptativo aos gráficos de $n p, u$ e $p$, e a Seção 7 resume as conclusões do trabalho.

\section{Gráfico Adaptativo de $c$}

Considere um processo de produção, do qual deseja-se monitorar a freqüência de nãoconformidades. Seja $x_{k}$ o número de não-conformidades da $k$-ésima amostra, composta de $m$ unidades de inspeção. Assume-se que $x_{k}$ siga uma distribuição de Poisson com média $c=m u$, onde $u$ é o número médio de não-conformidades por unidade de inspeção, e que não exista correlação serial entre amostras. Considera-se que o processo inicie sua operação em controle com $u=u_{0}$ mas, que após a ocorrência de uma causa especial, ele mude para um estado fora de controle, que faça a freqüência de não-conformidades $u$ aumentar 
imediatamente de $u_{0}$ para $u_{1}$. O processo permanece fora de controle até que a causa especial seja detectada e subseqüentemente eliminada, trazendo o processo de volta ao estado em controle. Suponha que um gráfico adaptativo de $c$ seja definido através de dois conjuntos de parâmetros, $\Theta_{1}=\left\{m_{1}, h_{1}, L S C_{1}, L S A_{1}\right\}$ e $\Theta_{2}=\left\{m_{2}, h_{2}, L S C_{2}, L S A_{2}\right\}$, onde $m_{i}$ é o tamanho de amostra, $h_{i}$ é o intervalo de tempo entre amostras, $L S C_{i}$ é o limite superior de controle e $L S A_{i}$ é o limite de advertência $(i=1,2)$. O gráfico é construído sem limite inferior de controle $(L I C)$, pois o interesse é detectar apenas ocorrências de deteriorações no processo que venham aumentar o número esperado de não-conformidades durante a produção. Assume-se, por definição, que $0<L S A_{i}<L S C_{i}(i=1,2), m_{1} \leq m_{2}$ e $h_{1} \geq h_{2}$ (no esquema $V p$, estas duas últimas desigualdades são estritas; a igualdade ocorre nos esquemas em que nem todos os parâmetros variam: VSS e $V S I$, por exemplo). A operação do gráfico adaptativo é simples e o esquema das amostragens obedece à seguinte regra: se $x_{k}<L S A_{i}$, para a $(k+1)$ ésima amostragem usa-se o conjunto de parâmetros $\Theta_{1}$; se $L S A_{i}<x_{k}<L S C_{i}$, usa-se o conjunto $\Theta_{2}$; se $x_{k}>L S C_{i}$ (alarme), inicia-se a investigação da causa especial. Para evitar gráficos com quatro limites $\left(L S C_{1}, L S C_{2}, L S A_{1}\right.$ e $\left.L S A_{2}\right)$, são utilizadas duas escalas verticais: uma à direita e outra à esquerda do gráfico. As escalas são lineares por partes, de modo que os $L S C$ 's coincidam em uma única linha e que o mesmo ocorra com os LSA's (ver Figura 1). Quando está em uso o conjunto $\Theta_{1}$, considera-se a escala da esquerda; quando está em uso o conjunto $\Theta_{2}$, considera-se a escala da direita.

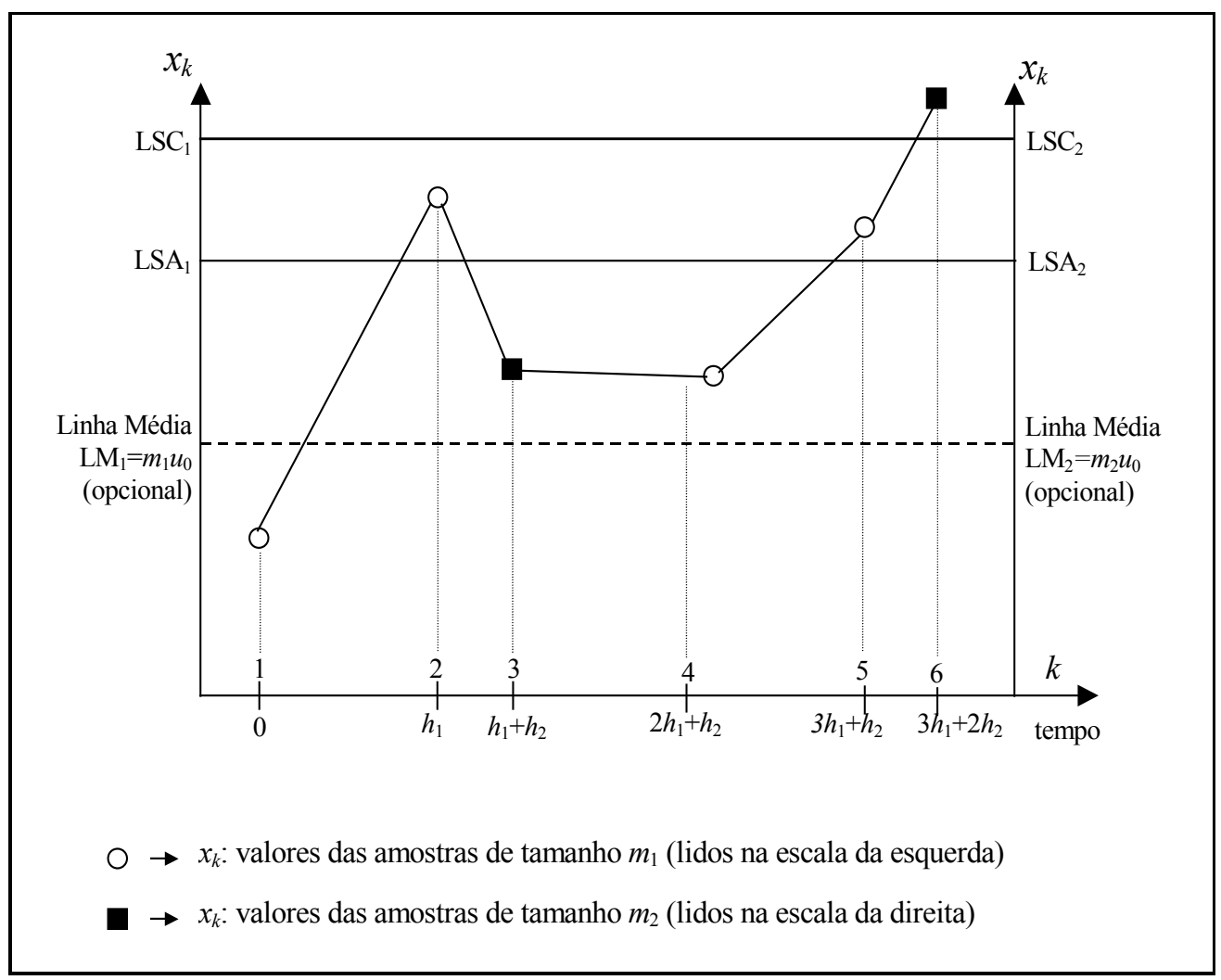

Figura 1 - Gráfico adaptativo (Vp) de $c$ com duas escalas. 
O princípio básico das regras de operação do gráfico totalmente adaptativo é simples: se $x_{k}$ encontra-se abaixo do limite de advertência, o controle é relaxado porque espera-se um tempo maior do que o usual para retirada da $(k+1)$ ésima amostra, de tamanho menor que o usual. Além disso, o valor de $x_{k+1}$ é registrado considerando a escala da esquerda, onde o limite superior de controle é maior que o usual. Por outro lado, se $x_{k}$ encontra-se entre o limite superior de controle e o limite de advertência, o controle se torna mais severo porque espera-se um tempo menor do que o usual para retirada da $(k+1)$ ésima amostra, de tamanho maior que o usual, e o valor de $x_{k+1}$ é registrado considerando a escala da direita, onde o limite superior de controle é mais estreito que o usual.

\section{Medidas de Desempenho}

O propósito do esquema adaptativo é acelerar a detecção, pelo gráfico, de incrementos no número médio de não-conformidades, sem precisar aumentar a quantidade de itens inspecionados e tampouco a taxa de alarmes falsos, que podem ser vistos como restrições preestabelecidas.

A velocidade de detecção pelo gráfico pode ser medida pelo tempo esperado entre a ocorrência de uma causa especial e sua sinalização (TES, "tempo esperado até o sinal" - ver Costa et al., 2004). As restrições relativas à quantidade média de itens a inspecionar por unidade de tempo, bem como à taxa máxima de alarmes falsos que o usuário está disposto a aceitar, são expressas em termos do tamanho médio de amostra $\bar{m}$, do intervalo médio de tempo entre amostragens $\bar{h}$, e do tempo médio entre ocorrências de alarmes falsos (TMAF).

Através das propriedades das cadeias de Markov, tem sido possível obter medidas de desempenho dos gráficos adaptativos (ver, por exemplo, Prabhu et al., 1994; e Costa, 1997, 1998a). Os estados da cadeia relacionam-se à posição da amostra $x_{k}$ da maneira indicada na Tabela 1, onde o índice $i$ em $L S A_{i}$ e $L S C_{i}$ refere-se ao conjunto de parâmetros usado para a $k$-ésima amostra.

Tabela 1 - Modelo de cadeia de Markov para o processo.

\begin{tabular}{|c|c|c|}
\hline $\begin{array}{c}\text { Estado da } \\
\text { cadeia }\end{array}$ & $\begin{array}{c}\text { Posição de } \boldsymbol{x}_{\boldsymbol{k}} \\
\text { no gráfico }\end{array}$ & $\begin{array}{c}\text { Conjunto de parâmetros para a retirada } \\
\text { da }(\boldsymbol{k}+\mathbf{1}) \text { ésima amostra }\end{array}$ \\
\hline 1 & $x_{k} \leq L S A_{i}$ & $\Theta_{1}$ \\
\hline 2 & $L S A_{i}<x_{k} \leq L S C_{i}$ & $\Theta_{2}$ \\
\hline 3 & $x_{k}>L S C_{i}$ & $\begin{array}{c}\text { estado absorvente } \\
\text { (processo interrompido) }\end{array}$ \\
\hline
\end{tabular}

A matriz de probabilidades de transição é $\mathbf{P}^{(f)}=\left[\begin{array}{ccc}p_{11}^{(f)} & p_{12}^{(f)} & p_{13}^{(f)} \\ p_{21}^{(f)} & p_{22}^{(f)} & p_{23}^{(f)} \\ 0 & 0 & 1\end{array}\right]$, com

$$
p_{i 1}^{(f)}=\sum_{j=0}^{\operatorname{int}\left(L S A_{i}\right)} e^{-m_{i} u_{f}}\left(m_{i} u_{f}\right)^{j} / j !
$$




$$
\begin{aligned}
& p_{i 2}^{(f)}=\sum_{j=\operatorname{int}\left(L S A_{i}\right)+1}^{\operatorname{int}\left(L S C_{i}\right)} e^{-m_{i} u_{f}}\left(m_{i} u_{f}\right)^{j} / j ! \\
& p_{i 3}^{(f)}=1-p_{i 1}^{(f)}-p_{i 2}^{(f)}
\end{aligned}
$$

onde $i=1,2$, o índice $f=0,1$ especifica se o processo está em controle $(f=0)$ ou fora de controle $(f=1), u_{1}>u_{0}$ e $\operatorname{int}(Y)$ representa a parte inteira de $Y$.

As probabilidades de estado inicial para cada fase seguem modelos diferentes.

$\mathrm{Na}$ fase em controle, o estado inicial $(\mathrm{em} k=0)$ corresponde ao conjunto de parâmetros $\left(\Theta_{1}\right.$ ou $\Theta_{2}$ ) usado para a primeira amostra (quando o processo está sendo iniciado, ou reiniciado após um alarme falso). Epprecht \& Costa (2001) demonstraram que existe um valor particular $r_{1}$ para a probabilidade de o estado inicial ser o estado 1 , tal que $r_{1}$ é a freqüência relativa esperada de visitas ao estado 1 . A expressão para $r_{1}$ depende do esquema adaptativo considerado. Para o gráfico VSI "puro", com limites constantes, $r_{1}$ é dado por

$$
r_{1}=p_{21}^{(0)} /\left(1-p_{11}^{(0)}-p_{23}^{(0)}+p_{21}^{(0)}\right)
$$

para todos os demais esquemas ( $V p, V S S, V S I L, V L), r_{1}$ é dado por

$$
r_{1}=\left(-b+\sqrt{b^{2}-4 a c}\right) /(2 a)
$$

onde

$$
a=p_{23}^{(0)}-p_{13}^{(0)}>0, \quad b=p_{12}^{(0)}+p_{21}^{(0)}+p_{13}^{(0)}-p_{23}^{(0)}, \quad \text { e } \quad c=-p_{21}^{(0)} .
$$

Para facilitar a modelagem, é conveniente assumir que o conjunto de parâmetros para a primeira amostra é escolhido aleatoriamente, $\Theta_{1}$ com probabilidade $r_{1}$ e $\Theta_{2}$ com probabilidade $r_{2}=\left(1-r_{1}\right)$.

Na prática, o usuário pode preferir adotar para a primeira amostra sempre o conjunto $\Theta_{1}$ ou o conjunto $\Theta_{2}$ em vez de sortear um deles. Se o processo permanecer tempo suficiente em controle, a escolha não-aleatória terá muito pouca influência nas propriedades do gráfico.

O conjunto de parâmetros para a primeira amostra após a ocorrência da causa especial (que, instantaneamente, aumenta $u$ de $u_{0}$ para $u_{1}$ ) será $\Theta_{1}$ se a causa especial ocorrer durante um intervalo de tempo entre amostras de duração $h_{1}$, e será $\Theta_{2}$ se ela ocorrer durante um intervalo de duração $h_{2}$. Esse conjunto de parâmetros corresponde ao estado inicial na fase fora de controle. Como em Reynolds et al. (1988), considera-se que $s_{i}$, a probabilidade de uma causa especial ocorrer durante um intervalo de duração $h_{i}$, é proporcional ao produto $r_{i} h_{i}$, sendo $r_{i}$ a freqüência relativa esperada de intervalos de duração $h_{i}$ até a ocorrência de uma causa especial.

$$
s_{i}=r_{i} h_{i} / \mathbf{r}^{\prime} \mathbf{h}, \quad i=1,2, \quad \mathbf{r}^{\prime}=\left[\begin{array}{ll}
r_{1} & r_{2}
\end{array}\right], \quad \mathbf{h}^{\prime}=\left[\begin{array}{ll}
h_{1} & h_{2}
\end{array}\right] .
$$

A teoria das cadeias de Markov possibilita a obtenção das expressões das medidas de desempenho de interesse. Desta forma, $N M A F_{\text {adapt }}=\mathbf{r}^{\prime} \mathbf{N}_{\mathbf{0}} \mathbf{1}$ é o número esperado de amostras até um alarme falso, e $N M A_{\text {adapt }}=\mathbf{s}^{\prime} \mathbf{N}_{1} \mathbf{1}$ é o número esperado de amostras até o sinal verdadeiro (na fase fora de controle), onde $\mathbf{s}^{\prime}=\left[\begin{array}{ll}s_{1} & s_{2}\end{array}\right], \mathbf{1}^{\prime}=\left[\begin{array}{ll}1 & 1\end{array}\right]$ e ambas as matrizes $\mathbf{N}_{\mathbf{f}}$ obedecem à fórmula geral 


$$
\mathbf{N}_{\mathbf{f}}=\left(\mathbf{I}-\mathbf{Q}_{\mathbf{f}}\right)^{-1} \quad(f=0,1)
$$

onde $\mathbf{Q}_{\mathbf{f}}=\left[\begin{array}{ll}p_{11}^{(f)} & p_{12}^{(f)} \\ p_{21}^{(f)} & p_{22}^{(f)}\end{array}\right]$, o que resulta em

$$
\mathbf{N}_{\mathbf{f}}=\frac{1}{\left(1-p_{11}^{(f)}\right)\left(1-p_{22}^{(f)}\right)-p_{12}^{(f)} p_{21}^{(f)}}\left[\begin{array}{cc}
\left(1-p_{22}^{(f)}\right) & p_{12}^{(f)} \\
p_{21}^{(f)} & \left(1-p_{11}^{(f)}\right)
\end{array}\right] \text {. }
$$

Além disso, $T M A F_{\text {adapt }}=\mathbf{r}^{\prime} \mathbf{N}_{\mathbf{0}} \mathbf{h}$ fornece o tempo médio até um alarme falso e

$$
T E S_{\text {adapt }}=\mathbf{s}^{\prime} \mathbf{N}_{\mathbf{1}} \mathbf{h}-\mathrm{E}(t)
$$

é o tempo para o gráfico sinalizar com o processo fora de controle, sendo $t$ o tempo entre a última amostra retirada durante o período em controle e o instante de ocorrência da causa especial. Como em Reynolds et al. (1988), supõe-se que, quando a causa especial ocorre dentro do intervalo de tempo entre duas amostras particulares quaisquer, o instante exato da ocorrência é uniformemente distribuído nesse intervalo. Conseqüentemente,

$$
\mathrm{E}(t)=\mathbf{s}^{\prime} \mathbf{h} / 2
$$

e, substituindo (4) em (3), TES $S_{\text {adapt }}=\mathbf{s}^{\prime} \mathbf{N}_{\mathbf{1}} \mathbf{h}-\mathbf{s}^{\prime} \mathbf{h} / 2$.

O tamanho médio de amostra na fase em controle é $\bar{m}=\left(\mathbf{r}^{\prime} \mathbf{N}_{\mathbf{0}} \mathbf{m}\right) / N M A F_{\text {adapt }}$, onde $\mathbf{m}^{\prime}=\left[\begin{array}{ll}m_{1} & m_{2}\end{array}\right]$, e o intervalo médio de tempo entre amostras na fase em controle é $\bar{h}=T M A F_{\text {adapt }} / N M A F_{\text {adapt }}$.

\section{Comparação entre Gráficos Adaptativos e Gráficos de Parâmetros Fixos}

Para comparar o desempenho dos diferentes esquemas adaptativos, os valores de TES de diversos projetos de gráficos de $c$ adaptativos e convencionais $(F p)$ foram obtidos para processos com diferentes valores de de $u_{0}$ e de $u_{1}$. Os resultados estão na Tabela 3. Para os gráficos $F p$, assume-se, sem perda de generalidade, $m=1$ e portanto $c_{0}=u_{0}$. A Tabela 2 apresenta os valores dos parâmetros desses gráficos. Note que esta tabela contém todos os tipos possíveis de esquemas adaptativos duais - i.e., em que um, dois ou todos os parâmetros do gráfico variam entre dois valores: $V p$, com todos os parâmetros variáveis; $V S S$, com $h_{1}=h_{2} ; V S I L, \operatorname{com} m_{1}=m_{2} ; V L, \operatorname{com} h_{1}=h_{2}$ e $m_{1}=m_{2} ;$ e $V S I$, com $m_{1}=m_{2}$ e $L S C_{1}=L S C_{2}$. (Relembrando, no caso de gráficos de Shewhart para atributos, não existe um esquema VSSI distinto do esquema $V p$, pois nele não se consegue manter fixo o risco de alarme falso, já que a função distribuição de probabilidades acumulada das variáveis aleatórias discretas é descontínua). A importância de considerar os esquemas em que nem todos os parâmetros são variáveis vem do fato de que, em muitos contextos práticos, pode não ser possível trabalhar com tamanho de amostra variável, ou com intervalo de tempo entre amostras variável.

A comparação é feita em igualdade de condições: na fase em controle do processo, os gráficos adaptativos considerados possuem, em média, mesmo tamanho de amostra, mesmo intervalo de tempo entre amostras e taxa de alarmes falsos não maior que dos gráficos $F p$ de 
referência. Formalmente, $\bar{h}=h$ e $\bar{m}=m$, onde $h$ e $m$ são, respectivamente, o intervalo de tempo entre amostras e o tamanho de amostra do gráfico $F p$.

Para o gráfico com parâmetros fixos, o TMAF e o TES são dados respectivamente por

$$
\begin{aligned}
& T M A F_{F p}=N M A F_{F p} \times h=h / \alpha \\
& T E S_{F p}=N M A_{F p} \times h-h / 2=[1 /(1-\beta)-1 / 2] h \\
& \operatorname{com} \alpha=1-\sum_{j=0}^{\operatorname{int}(L S C)} e^{-m u_{0}}\left(m u_{0}\right)^{j} / j ! \text { e } \beta=\sum_{j=0}^{\operatorname{int}(L S C)} e^{-m u_{1}}\left(m u_{1}\right)^{j} / j ! ; \operatorname{int}(Y) \text { representa a parte }
\end{aligned}
$$

inteira de $Y$.

A função de distribuição do número de não-conformidades na amostra, sendo descontínua, não permite satisfazer simultaneamente as condições $m=\bar{m}, h=\bar{h}$ e $T M A F_{\text {adapt }}=T M A F_{F p}$. Por isso, esta última restrição será substituída por $T M A F_{a d a p t} \geq T M A F_{F p}$. Além disso, sem perda de generalidade, adotou-se para o gráfico de parâmetros fixos $h=1$ e $m=1$, e, para o gráfico adaptativo, $\bar{h}=1$ e $\bar{m}=1$; isso corresponde a empregar, como unidade de tempo, o intervalo de tempo entre amostras do gráfico $F p$ e, como unidade de inspeção, o tamanho de amostra do gráfico $F p$.

Os projetos da Tabela 2 foram selecionados satisfazendo os seguintes critérios:

No caso dos gráficos $F p$, em que o projeto limita-se à escolha do $L S C$, foi adotado o menor valor de $L S C$ que ainda satisfaz à condição $T M A F_{F p} \geq 200-$ limite que nem sempre coincide com o tradicional limite "de três sigma". Isso porque, com valores de $c_{0}<9$, como é o caso de interesse aqui, o limite "de três sigma" leva por vezes a valores de TMAF inaceitavelmente baixos; torna-se portanto recomendável determinar o $L S C$ em função da taxa de alarmes falsos tolerada. Por outro lado, se essa taxa fosse fixada no valor convencional $\alpha=0,0027$ (que é a taxa "nominal" dos gráficos de Shewhart convencionais quando a estatística amostral plotada é normalmente distribuída), os valores de $L S C$ resultantes seriam muito altos, enfraquecendo o poder do gráfico de detectar desvios no processo. $\mathrm{O}$ valor $T M A F_{F p} \geq 200$ foi considerado uma boa solução de compromisso. Esse valor também é reportado por Bissel (1988) como um valor tradicional: "For attribute and event situations, because of the reduced sensitivity of discrete variables to changes in the process, the Action lines have traditionally been set at the upper $0.5 \%$ (1 in 200) point of the approximate Binomial or Poisson distribution".

No caso dos gráficos adaptativos: cada projeto é o que minimiza o TES quando $u$ dobra (i.e., para $u_{1}=2 u_{0}$ ), com as restrições já mencionadas $\bar{h}=h, \bar{m}=m$ e $T M A F_{\text {adapt }} \geq$ $T M A F_{F p}$ (em relação ao gráfico $F p$ para o mesmo valor de $u_{0}=c_{0}$ ). Estes projetos são os fornecidos por Epprecht et al. (2003), que os obtiveram por busca exaustiva no espaço dos parâmetros $\Theta_{1}$ e $\Theta_{2}$ (acrescentando as restrições $0,2<m_{1}<0,8$ e $m_{2}<5,0$ por considerações de ordem prática). No caso dos esquemas Vp, VSIL e VSI, foram consideradas apenas duas opções para o menor intervalo de amostragem $h_{2}: 0,10$ e 0,50 . Isso devido ao fato de nem todo valor de $h_{2}$ ser factível na prática, e também para indicar a sensibilidade dos resultados em relação a $h_{2} ; 0$ intervalo $h_{l}$ foi determinado univocamente pela restrição $\bar{h}=h$. 
Tabela 2 - Parâmetros de projeto para o gráfico tradicional e para gráficos adaptativos de $c$.

\begin{tabular}{|c|c|c|c|c|c|c|c|c|c|}
\hline$c_{0}=u_{0}$ & Esquema & $h_{2}$ & $h_{1}$ & $m_{1}$ & $m_{2}$ & $L S C_{1}$ & $L S A_{1}$ & $L S C_{2}$ & $L S A_{2}$ \\
\hline \multirow[t]{9}{*}{0,5} & $\mathrm{Fp}$ & 1 & 1 & 1 & 1 & 3,5 & ---- & 3,5 & ---- \\
\hline & $\mathrm{Vp}$ & 0,1 & 1,180 & 0,256 & 4,615 & 3,5 & 0,5 & 6,5 & 2,5 \\
\hline & $\mathrm{Vp}$ & 0,5 & 1,100 & 0,256 & 4,615 & 3,5 & 0,5 & 6,5 & 2,5 \\
\hline & VSS & 1 & 1 & 0,256 & 4,615 & 3,5 & 0,5 & 6,5 & 2,5 \\
\hline & VSIL & 0,1 & 1,192 & 1 & 1 & 5,5 & 1,5 & 2,5 & 0,5 \\
\hline & VSIL & 0,5 & 1,133 & 1 & 1 & 5,5 & 1,5 & 2,5 & 0,5 \\
\hline & VL & 1 & 1 & 1 & 1 & 4,5 & 1,5 & 2,5 & 1,5 \\
\hline & VSI & 0,1 & 1,581 & 1 & 1 & 3,5 & 0,5 & 3,5 & 0,5 \\
\hline & VSI & 0,5 & 1,323 & 1 & 1 & 3,5 & 0,5 & 3,5 & 0,5 \\
\hline \multirow[t]{9}{*}{1,0} & $\mathrm{Fp}$ & 1 & 1 & 1 & 1 & 4,5 & ---- & 4,5 & ---- \\
\hline & $\mathrm{Vp}$ & 0,1 & 1,317 & 0,256 & 3,075 & 3,5 & 0,5 & 7,5 & 3,5 \\
\hline & $\mathrm{Vp}$ & 0,5 & 1,176 & 0,256 & 3,075 & 3,5 & 0,5 & 7,5 & 3,5 \\
\hline & VSS & 1 & 1 & 0,208 & 4,195 & 2,5 & 0,5 & 9,5 & 5,5 \\
\hline & VSIL & 0,1 & 1,222 & 1 & 1 & 5,5 & 2,5 & 3,5 & 0,5 \\
\hline & VSIL & 0,5 & 1,140 & 1 & 1 & 5,5 & 2,5 & 3,5 & 0,5 \\
\hline & VL & 1 & 1 & 1 & 1 & 6,5 & 2,5 & 3,5 & 0,5 \\
\hline & VSI & 0,1 & 2,538 & 1 & 1 & 4,5 & 0,5 & 4,5 & 0,5 \\
\hline & VSI & 0,5 & 1,854 & 1 & 1 & 4,5 & 0,5 & 4,5 & 0,5 \\
\hline \multirow[t]{9}{*}{1,5} & $\mathrm{Fp}$ & 1 & 1 & 1 & 1 & 5,5 & ---- & 5,5 & ---- \\
\hline & $\mathrm{Vp}$ & 0,1 & 1,258 & 0,528 & 2,620 & 5,5 & 1,5 & 8,5 & 4,5 \\
\hline & $\mathrm{Vp}$ & 0,5 & 1,122 & 0,504 & 3,005 & 4,5 & 1,5 & 9,5 & 5,5 \\
\hline & VSS & 1 & 1 & 0,216 & 2,970 & 5,5 & 0,5 & 9,5 & 5,5 \\
\hline & VSIL & 0,1 & 1,250 & 1 & 1 & 7,5 & 3,5 & 4,5 & 0,5 \\
\hline & VSIL & 0,5 & 1,210 & 1 & 1 & 6,5 & 3,5 & 4,5 & 0,5 \\
\hline & VL & 1 & 1 & 1 & 1 & 7,5 & 3,5 & 4,5 & 0,5 \\
\hline & VSI & 0,1 & 4,116 & 1 & 1 & 5,5 & 0,5 & 5,5 & 0,5 \\
\hline & VSI & 0,5 & 2,731 & 1 & 1 & 5,5 & 0,5 & 5,5 & 0,5 \\
\hline \multirow[t]{9}{*}{2,0} & $\mathrm{Fp}$ & 1 & 1 & 1 & 1 & 6,5 & ---- & 6,5 & ---- \\
\hline & $\mathrm{Vp}$ & 0,1 & 1,254 & 0,432 & 3,005 & 6,5 & 1,5 & 11,5 & 7,5 \\
\hline & $\mathrm{Vp}$ & 0,5 & 1,141 & 0,432 & 3,005 & 6,5 & 1,5 & 11,5 & 7,5 \\
\hline & VSS & 1 & 1 & 0,392 & 3,635 & 4,5 & 1,5 & 13,5 & 9,5 \\
\hline & VSIL & 0,1 & 1,308 & 1 & 1 & 8,5 & 3,5 & 5,5 & 1,5 \\
\hline & VSIL & 0,5 & 1,172 & 1 & 1 & 8,5 & 3,5 & 5,5 & 1,5 \\
\hline & VL & 1 & 1 & 1 & 1 & 7,5 & 4,5 & 4,5 & 2,5 \\
\hline & VSI & 0,1 & 2,307 & 1 & 1 & 6,5 & 1,5 & 6,5 & 1,5 \\
\hline & VSI & 0,5 & 1,726 & 1 & 1 & 6,5 & 1,5 & 6,5 & 1,5 \\
\hline \multirow[t]{9}{*}{3,0} & $\mathrm{Fp}$ & 1 & 1 & 1 & 1 & 8,5 & ---- & 8,5 & ---- \\
\hline & $\mathrm{Vp}$ & 0,1 & 1,174 & 0,467 & 3,717 & 6,5 & 2,5 & 18,5 & 14,5 \\
\hline & $\mathrm{Vp}$ & 0,5 & 1,097 & 0,467 & 3,717 & 6,5 & 2,5 & 18,5 & 14,5 \\
\hline & VSS & 1 & 1 & 0,467 & 3,717 & 6,5 & 2,5 & 18,5 & 14,5 \\
\hline & VSIL & 0,1 & 1,385 & 1 & 1 & 10,5 & 4,5 & 7,5 & 2,5 \\
\hline & VSIL & 0,5 & 1,215 & 1 & 1 & 10,5 & 4,5 & 7,5 & 2,5 \\
\hline & VL & 1 & 1 & 1 & 1 & 9,5 & 6,5 & 6,5 & 2,5 \\
\hline & VSI & 0,1 & 2,219 & 1 & 1 & 8,5 & 2,5 & 8,5 & 2,5 \\
\hline & VSI & 0,5 & 1,677 & 1 & 1 & 8,5 & 2,5 & 8,5 & 2,5 \\
\hline
\end{tabular}




\begin{tabular}{cccccccccc}
\hline 4,0 & Fp & 1 & 1 & 1 & 1 & 10,5 & ---- & 10,5 & ---- \\
& Vp & 0,1 & 1,232 & 0,600 & 2,550 & 8,5 & 3,5 & 18,5 & 13,5 \\
& Vp & 0,5 & 1,152 & 0,440 & 2,783 & 7,5 & 2,5 & 19,5 & 14,5 \\
& VSS & 1 & 1 & 0,440 & 2,783 & 7,5 & 2,5 & 19,5 & 14,5 \\
& VSIL & 0,1 & 1,450 & 1 & 1 & 12,5 & 5,5 & 9,5 & 3,5 \\
& VSIL & 0,5 & 1,105 & 1 & 1 & 11,5 & 7,5 & 8,5 & 3,5 \\
& VL & 1 & 1 & 1 & 1 & 11,5 & 7,5 & 8,5 & 4,5 \\
& VSI & 0,1 & 2,170 & 1 & 1 & 10,5 & 3,5 & 10,5 & 3,5 \\
& VSI & 0,5 & 1,650 & 1 & 1 & 10,5 & 3,5 & 10,5 & 3,5 \\
\hline
\end{tabular}

Para cada combinação de valores de $u_{0}=c_{0}$ e de $\gamma=u_{1} / u_{0}$, o menor TES para os diferentes gráficos encontra-se destacado em negrito na Tabela 3. E, para as situações em que não seja viável na prática adotar $h_{2}$ igual a 1/10 do intervalo de tempo "unitário", estão sublinhados os menores valores de TES dentre os dos gráficos que usam $h_{2}=0,50$ ou $h$ fixo (para cada combinação de valores de $u_{0}=c_{0}$ e de $\gamma=u_{1} / u_{0}$ ).

As seguintes observações podem ser tiradas da Tabela 3:

Para todos os valores de $c_{0}$ considerados, o esquema $V p$ fornece tempos médios de sinalização de aumentos da ordem de $50 \%$ a $200 \%$ em $u$ (i.e., valores de $T E S$ para $1,5 u_{0} \leq$ $u_{1} \leq 3 u_{0}$ ) que são duas a cinco vezes menores que os dos gráficos $F p$ correspondentes.

Para $0,5 \leq c_{0} \leq 1,5$, o esquema $V p$ é o mais eficiente de todos para sinalizar aumentos de até 3 vezes em $u\left(\gamma \leq 3,0\right.$; e inclusive para $\gamma \leq 4,0$ no caso de $\left.c_{0}=0,5\right)$. Para $2,0 \leq c_{0} \leq 4,0$, o esquema $V p$ ainda é o mais eficiente de todos para sinalizar aumentos de até 2 vezes em $u(\gamma \leq 2,0)$. Para aumentos maiores em $u$ (quando o esquema $V p$ deixa de ser o mais eficiente), o esquema mais eficiente é o esquema $V S I L$, até o ponto em que o esquema $F p$ se torna o mais eficiente (o que ocorre a partir de $u_{1}=5 u_{0}$, para $c_{0}=2,0$; e a partir de $u_{1}=4 u_{0}$, para $c_{0} \geq 3,0$ ).

O esquema $V S S$ é o segundo melhor esquema para valores pequenos de $c_{0}$ e pequenos aumentos em $u_{0}$ (especificamente, para $c_{0}=0,5$ e $\gamma \leq 3,5$; para $1,0 \leq c_{0} \leq 1,5$ e $\gamma \leq 2,0$; e para $2,0 \leq c_{0} \leq 3,0$ e $\left.\gamma=1,5\right)$; nos demais casos, em geral o segundo melhor esquema é o esquema $V S I L$.

Nos esquemas em que $h$ é variável ( $V p, V S I L$ e $V S I)$, os melhores resultados foram obtidos com os menores valores de $h_{2}$.

Assim, os resultados mencionados dos esquemas Vp, VSIL e VSI referem-se ao seu emprego com $h_{2}=0,10$ (um décimo do intervalo de tempo base, "unitário", igual por hipótese ao intervalo de amostragem do gráfico $F p$ de referência para comparação). Pode ser feita uma análise semelhante, considerando apenas o uso de $h_{2}=0,50$ ou $h$ fixo.

Em geral o esquema VSIL é superior ao esquema VSI. O esquema adaptativo com o mais fraco desempenho é o esquema VL, mas mesmo assim ele apresenta ganhos significativos em relação ao esquema $F p$ para casos $\operatorname{com} \gamma \leq 3,0$.

Sintetizando as duas últimas observações, pode-se dizer que, como regra geral, da mesma maneira que ocorre com a aplicação de esquemas adaptativos a gráficos de controle por variáveis (ver por exemplo Costa, 1999a), esquemas adaptativos de maior ordem (com maior número de parâmetros variando entre dois valores) são mais sensíveis para a detecção de alterações pequenas a moderadas no nível de qualidade do processo; além disso, deve-se adotar o menor valor operacionalmente possível para $h_{2}$. 
Epprecht et al. (2003) calcularam também os desvios-padrão dos tempos até a sinalização, mostrando que seus valores são muito próximos aos valores de TES. Os valores não são aqui reproduzidos, por limitação de espaço; a conclusão importante de ordem prática é que a análise dos valores de TES é suficiente para comparação dos desempenhos dos esquemas.

Tabela 3 - Valores de TES para o gráfico tradicional e para gráficos adaptativos de $c$.

\begin{tabular}{|c|c|c|c|c|c|c|c|c|c|c|c|}
\hline \multirow[b]{2}{*}{$c_{0}=u_{0}$} & \multirow[b]{2}{*}{ Esquema } & \multirow[b]{2}{*}{$h_{2}$} & \multirow[b]{2}{*}{ TMAF } & \multicolumn{8}{|c|}{$\gamma=u_{1} / u_{0}$} \\
\hline & & & & 1,5 & 2,0 & 2,5 & 3,0 & 3,5 & 4,0 & 4,5 & $\mathbf{5 , 0}$ \\
\hline \multirow[t]{9}{*}{0,5} & $\mathrm{Fp}$ & & 570,9 & 136,6 & 52,16 & 25,63 & 14,73 & 9,42 & 6,50 & 4,75 & 3,63 \\
\hline & $\mathrm{Vp}$ & 0,1 & 628,2 & 43,13 & 9,66 & 4,82 & 3,46 & 2,84 & 2,46 & 2,19 & 1,98 \\
\hline & $\mathrm{Vp}$ & 0,5 & 628,2 & $\underline{46,33}$ & $\underline{10,91}$ & $\underline{5,38}$ & $\underline{3,76}$ & $\underline{3,04}$ & $\underline{2,62}$ & $\underline{2,34}$ & $\underline{2,14}$ \\
\hline & VSS & & 628,2 & $\overline{50,36}$ & $\overline{12,49}$ & $\overline{6,09}$ & $\overline{4,15}$ & $\overline{3,29}$ & $\overline{2,82}$ & $\overline{2,52}$ & $\overline{2,32}$ \\
\hline & VSIL & 0,1 & 571,8 & 88,08 & 25,51 & 10,66 & 5,66 & 3,55 & 2,50 & 1,91 & 1,55 \\
\hline & VSIL & 0,5 & 571,4 & 93,32 & 29,03 & 13,02 & 7,35 & 4,82 & 3,50 & 2,73 & 2,24 \\
\hline & VL & & 696,5 & 130,6 & 44,24 & 20,62 & 11,70 & 7,55 & 5,34 & 4,03 & 3,19 \\
\hline & VSI & 0,1 & 570,9 & 110,3 & 34,63 & 14,36 & 7,22 & 4,21 & 2,76 & 2,01 & 1,58 \\
\hline & VSI & 0,5 & 570,9 & 122,0 & 42,36 & 19,31 & 10,49 & 6,46 & 4,36 & 3,16 & 2,42 \\
\hline \multirow[t]{9}{*}{1,0} & $\mathrm{Fp}$ & & 273,2 & 53,33 & 18,49 & 8,69 & 4,91 & 3,14 & 2,19 & 1,64 & 1,29 \\
\hline & $\mathrm{Vp}$ & 0,1 & 273,7 & 18,19 & 4,55 & 2,56 & 1,95 & 1,66 & 1,47 & 1,33 & 1,23 \\
\hline & $\mathrm{Vp}$ & 0,5 & 273,7 & $\underline{20,13}$ & $\underline{5,26}$ & $\underline{2,86}$ & $\underline{2,12}$ & $\underline{1,78}$ & 1,58 & 1,45 & 1,36 \\
\hline & VSS & & 312,2 & 24,58 & 6,12 & 3,29 & 2,45 & 2,08 & 1,88 & 1,74 & 1,64 \\
\hline & VSIL & 0,1 & 273,5 & 26,74 & 6,99 & 3,20 & 1,97 & 1,43 & 1,14 & $\mathbf{0 , 9 7}$ & 0,86 \\
\hline & VSIL & 0,5 & 273,7 & 30,21 & 8,90 & 4,33 & 2,71 & 1,94 & $\underline{1,51}$ & $\underline{1,24}$ & $\underline{1,06}$ \\
\hline & VL & & 298,2 & 36,18 & 11,74 & 6,01 & 3,84 & 2,78 & 2,17 & 1,78 & 1,51 \\
\hline & VSI & 0,1 & 273,2 & 35,75 & 9,26 & 3,85 & 2,29 & 1,72 & 1,48 & 1,36 & 1,30 \\
\hline & VSI & 0,5 & 273,2 & 43,40 & 13,19 & 5,83 & 3,29 & 2,18 & 1,63 & 1,31 & 1,12 \\
\hline \multirow[t]{9}{*}{1,5} & $\mathrm{Fp}$ & & 224,4 & 36,04 & 11,42 & 5,15 & 2,87 & 1,84 & 1,30 & 1,00 & 0,82 \\
\hline & $\mathrm{Vp}$ & 0,1 & 225,1 & 11,62 & 2,99 & 1,73 & 1,31 & 1,10 & 0,97 & 0,88 & 0,82 \\
\hline & $\mathrm{Vp}$ & 0,5 & 234,0 & $\underline{13,57}$ & $\underline{3,54}$ & $\underline{2,03}$ & $\underline{1,53}$ & $\underline{1,28}$ & 1,13 & 1,02 & 0,94 \\
\hline & VSS & & 224,5 & 15,00 & 4,15 & 2,38 & 1,84 & 1,62 & 1,51 & 1,44 & 1,40 \\
\hline & VSIL & 0,1 & 241,5 & 15,67 & 4,29 & 2,21 & 1,47 & 1,12 & $\mathbf{0 , 9 3}$ & $\mathbf{0 , 8 2}$ & 0,75 \\
\hline & VSIL & 0,5 & 224,5 & 18,73 & 5,75 & 2,98 & 1,93 & 1,42 & $\underline{1,12}$ & $\underline{0,94}$ & $\underline{0,82}$ \\
\hline & VL & & 241,2 & 23,02 & 7,68 & 4,06 & 2,63 & 1,92 & 1,49 & 1,22 & 1,03 \\
\hline & VSI & 0,1 & 224,42 & 20,92 & 5,38 & 2,90 & 2,29 & 2,08 & 2,00 & 1,96 & 1,94 \\
\hline & VSI & 0,5 & 224,42 & 27,30 & 7,71 & 3,55 & 2,20 & 1,63 & 1,34 & 1,19 & 1,09 \\
\hline \multirow[t]{9}{*}{2,0} & $\mathrm{Fp}$ & & 220,6 & 29,34 & 8,54 & 3,70 & 2,04 & 1,32 & 0,96 & $\underline{0,76}$ & $\underline{0,65}$ \\
\hline & $\mathrm{Vp}$ & 0,1 & 222,1 & 8,65 & 2,35 & 1,48 & 1,17 & 1,01 & 0,91 & 0,84 & 0,79 \\
\hline & $\mathrm{Vp}$ & 0,5 & 222,1 & $\underline{9,68}$ & $\underline{2,72}$ & $\underline{1,71}$ & $\underline{1,38}$ & 1,21 & 1,12 & 1,04 & 0,99 \\
\hline & VSS & & 237,7 & 11,31 & 3,17 & 2,00 & 1,62 & 1,42 & 1,29 & 1,18 & 1,08 \\
\hline & VSIL & 0,1 & 228,0 & 12,61 & 2,99 & 1,50 & 1,05 & 0,86 & 0,77 & 0,72 & 0,69 \\
\hline & VSIL & 0,5 & 228,0 & 15,73 & 4,32 & 2,19 & 1,45 & $\underline{1,11}$ & $\underline{0,92}$ & 0,79 & 0,71 \\
\hline & VL & & 221,2 & 19,14 & 5,56 & 2,78 & 1,79 & 1,30 & 1,02 & 0,84 & 0,72 \\
\hline & VSI & 0,1 & 220,6 & 17,09 & 3,72 & 1,78 & 1,34 & 1,20 & 1,14 & 1,12 & 1,10 \\
\hline & VSI & 0,5 & 220,6 & 22,39 & 5,71 & 2,49 & 1,51 & 1,11 & 0,92 & 0,81 & 0,76 \\
\hline
\end{tabular}




\begin{tabular}{|cccccccccccc|}
\hline 3,0 & Fp & & 263,0 & 24,34 & 6,05 & 2,46 & 1,34 & 0,89 & $\underline{\mathbf{0 , 6 8}}$ & $\underline{\mathbf{0 , 5 9}}$ & $\underline{\mathbf{0 , 5 4}}$ \\
& Vp & 0,1 & 265,8 & $\mathbf{6 , 0 8}$ & $\mathbf{1 , 8 3}$ & 1,22 & 0,97 & 0,83 & 0,74 & 0,69 & 0,66 \\
& Vp & 0,5 & 265,8 & $\underline{6,73}$ & $\underline{2,09}$ & $\underline{1,43}$ & 1,18 & 1,02 & 0,91 & 0,82 & 0,75 \\
& VSS & & 265,8 & 7,54 & 2,39 & 1,69 & 1,42 & 1,25 & 1,11 & 0,99 & 0,89 \\
& VSIL & 0,1 & 265,2 & 8,99 & 2,00 & $\mathbf{1 , 1 1}$ & $\mathbf{0 , 8 6}$ & $\mathbf{0 , 7 6}$ & 0,72 & 0,70 & 0,69 \\
& VSIL & 0,5 & 265,4 & 12,20 & 3,10 & 1,59 & $\underline{1,09}$ & $\underline{0,86}$ & 0,73 & 0,65 & 0,61 \\
& VL & & 306,4 & 14,87 & 4,15 & 2,09 & 1,33 & 0,96 & 0,75 & 0,63 & 0,57 \\
& VSI & 0,1 & 263,0 & 12,57 & 2,46 & 1,37 & 1,16 & 1,09 & 1,07 & 1,06 & 1,05 \\
& VSI & 0,5 & 263,0 & 17,66 & 3,92 & 1,72 & 1,10 & 0,87 & 0,76 & 0,71 & 0,69 \\
\hline 4,0 & Fp & & 352,1 & 22,96 & 4,93 & 1,90 & 1,03 & $\underline{0,71}$ & $\mathbf{0 , 5 8}$ & $\underline{\mathbf{0 , 5 3}}$ & $\mathbf{0 , 5 1}$ \\
& Vp & 0,1 & 404,5 & $\mathbf{6 , 3 0}$ & $\mathbf{1 , 3 8}$ & $\mathbf{0 , 9 1}$ & $\mathbf{0 , 7 7}$ & $\mathbf{0 , 7 0}$ & 0,66 & 0,64 & 0,62 \\
& Vp & 0,5 & 356,7 & $\underline{6,48}$ & $\underline{1,72}$ & $\underline{1,20}$ & 1,02 & 0,91 & 0,82 & 0,75 & 0,70 \\
& VSS & & 356,7 & 7,67 & 2,06 & 1,42 & 1,22 & 1,09 & 0,97 & 0,86 & 0,77 \\
& VSIL & 0,1 & 352,6 & 7,48 & 1,60 & 0,97 & 0,81 & 0,75 & 0,72 & 0,71 & 0,71 \\
& VSIL & 0,5 & 352,8 & 10,05 & 2,52 & 1,29 & 0,88 & 0,69 & 0,61 & 0,57 & 0,55 \\
& VL & & 419,1 & 14,31 & 3,43 & 1,67 & 1,05 & 0,77 & 0,62 & 0,55 & 0,52 \\
& VSI & 0,1 & 352,1 & 10,62 & 1,95 & 1,22 & 1,09 & 1,05 & 1,04 & 1,03 & 1,03 \\
& VSI & 0,5 & 352,1 & 15,97 & 3,14 & 1,39 & 0,93 & 0,77 & 0,70 & 0,68 & 0,67 \\
\hline
\end{tabular}

\section{Projeto dos Gráficos de $c$ com Parâmetros Variáveis}

A análise precedente mostrou uma maior eficiência dos gráficos adaptativos de $c$ em relação ao gráfico tradicional, de parâmetros fixos, para detectar aumentos em $u$ de até cerca de quatro vezes o valor em controle, em processos com $c_{0} \leq 4,0$. Um problema de ordem prática é a escolha do conjunto de valores para os parâmetros do gráfico adaptativo.

Em tese, os projetos constantes da Tabela 2 forneceriam a resposta a este problema. Na prática, porém, o problema permanece, pois:

A escolha dos valores para os parâmetros do gráfico depende dos valores de $u_{0}$ (freqüência de não-conformidades em controle do processo) e $u_{1}$ (valor da freqüência de não-conformidades contra o qual se deseja maior proteção). $\mathrm{O}$ valor de $u_{0}$ do processo em questão pode não ser nenhum dos valores contemplados na Tabela 2. Além disso, os projetos da Tabela 2 são os que minimizam o TES para $\gamma=2,0$ (i.e., para $u_{1}$ igual ao dobro de $u_{0}$ ); no entanto o usuário pode estar interessado em minimizar o TES para valores de $\gamma$ diferentes deste. Epprecht et al. (2003) obtiveram projetos ótimos para $\gamma=1,5 ; 3,0$. (Já a escolha do "melhor" projeto para uma faixa de valores de $\gamma$ seria um problema multicritério, encerrando um razoável grau de subjetividade).

E, mesmo supondo que o usuário esteja interessado no melhor projeto para um par de valores de $u_{0}$ e $\gamma$ contemplados na Tabela 2, essa tabela fornece valores para $h_{1}, m_{1}$ e $m_{2}$ que podem não ser praticáveis em muitas situações. Pode-se imaginar que os valores "operacionalizáveis" para o intervalo amostral pertençam a um domínio discreto, como por exemplo $\{30 \mathrm{~min}, 1 \mathrm{~h}, 2 \mathrm{~h}, 3 \mathrm{~h}, \ldots\}$, e que, da mesma forma, só haja um conjunto limitado de tamanhos de amostra admissíveis, múltiplos de alguma unidade básica, como por exemplo " 1 rolo" (prosseguindo com este exemplo, se $m=1$ corresponder a 5 rolos, os valores admissíveis para $m$ serão 0,$2 ; 0,4 ; 0,6 ; \ldots)$. 
O propósito primordial da Tabela 2 não foi fornecer parâmetros para implementação do esquema em cada situação prática, mas sim comparar os desempenhos dos esquemas para mostrar a superioridade do esquema adaptativo. De acordo com esse propósito, os valores pouco práticos para $h_{1}, m_{1}$ e $m_{2}$ nos projetos da Tabela 2 foram necessários para atender às restrições $T M A F_{\text {adapt }} \geq T M A F_{F p}, \bar{h} \geq h=1$ e $\bar{m} \leq m=1$ com a menor folga possível, com a finalidade de reduzir a comparação de eficiência entre esquemas a uma simples comparação de seus TES's. Se houvesse folga nas restrições, as diferenças entre os TES's diminuiriam e os ganhos em eficiência proporcionados pelos esquemas adaptativos ficariam diluídos entre um menor valor de TES, um menor tamanho médio de amostra, uma menor freqüência média de amostragem, e/ou uma maior proteção contra alarmes falsos. A visualização do ganho tornar-se-ia menos imediata, dificultando a comparação entre esquemas.

Ora, em geral, com $h_{1}, m_{1}$ e $m_{2}$ limitados a um pequeno conjunto de valores práticos, não seria possível atender às restrições sem folgas, uma vez que os parâmetros de projeto restantes (limites de controle e de advertência) não se prestam a um "ajuste fino" de $\bar{h}, \bar{m} \mathrm{e}$ $T M A F$, devido à natureza discreta das estatísticas de contagem.

Porém, na prática, as condições para escolha dos parâmetros do gráfico são outras. Se o usuário não estiver satisfeito com a eficiência dos gráficos de $c$ com parâmetros fixos para sinalizar um determinado aumento na freqüência média de não-conformidades do seu processo, o esquema adaptativo é uma alternativa mais eficiente. O problema do usuário não é demonstrar isso, mas escolher o "melhor" projeto adaptativo, segundo critérios de eficiência e operacionalidade. Nesse contexto, é mais importante usar valores operacionalmente convenientes para $h_{1}, h_{2}, m_{1}$ e $m_{2}$ do que satisfazer a $\bar{h}=h$ e $\bar{m}=m$. As condições a serem satisfeitas por $\bar{h}$ e $\bar{m}$ podem ser expressas por uma única restrição:

$$
\bar{m} / \bar{h} \leq m / h,
$$

ou seja, de que o custo de amostragem (supondo-o proporcional ao número médio de unidades inspecionadas por unidade de tempo) do gráfico adaptativo não seja superior ao do gráfico $F p$ de referência. Isso fornece uma maior flexibilidade, ampliando o conjunto de soluções factíveis. Além disso, uma pequena violação da restrição (5) pode ser tolerável, se ela resultar em uma grande melhora no TES. Para comparar diferentes projetos de gráficos levando em conta esse "tradeoff", pode-se usar a forma genérica da função $g$ recomendada por Epprecht \& Santos (1998):

$$
g=T E S \times(\bar{m} / \bar{h}) .
$$

onde, no caso de projetos $F p$, evidentemente, $\bar{m}=m$ e $\bar{h}=h$. Quando diferentes projetos para o gráfico possuírem o mesmo valor de $\bar{m} / \bar{h}$, o de menor $g$ terá o menor TES; quando diferentes projetos possuírem o mesmo valor de TES, o de menor $g$ terá a menor razão $\bar{m} / \bar{h}$. No caso mais geral, entre dois projetos com valores de TES e de $\bar{m} / \bar{h}$ diferentes, o projeto de menor $g$ será o mais eficiente, pois possui a maior razão (quociente) entre rapidez média de detecção (medida por 1/TES) e custo por unidade de tempo (medido por $\bar{m} / \bar{h}$ ). 
Assim, pode-se quantificar a eficiência relativa de um esquema A em relação a um esquema $\mathrm{B}$ como $g_{\mathrm{B}} / g_{\mathrm{A}}$, razão que será maior do que a unidade quando o esquema A for mais eficiente que o esquema $\mathrm{B}$.

Não é difícil escrever um programa para testar automaticamente todas as combinações admissíveis de valores para $h_{1}, h_{2}, m_{1}$ e $m_{2}$; experimentar (para cada uma delas) um conjunto de valores razoáveis para os limites de controle; calcular, para cada conjunto, as medidas de desempenho, pelas expressões fornecidas na Seção 3, descartando os projetos que não atendam a algumas restrições (por exemplo, em $T M A F$, ou em $T M A F$ e em $\bar{m} / \bar{h}$ ); de modo a, finalmente: ou selecionar dentre os projetos não eliminados aquele que minimize alguma função-objetivo (como o TES, ou $g=T E S \times(\bar{m} / \bar{h})$, dependendo das restrições consideradas); ou simplesmente, listar como saídas os projetos não eliminados, junto com suas medidas de desempenho, para escolha de um deles pelo usuário.

Contudo, se o número de valores admissíveis para $h_{1}, h_{2}, m_{1}$ e $m_{2}$ for pequeno (ainda mais considerando que nem todas as combinações desses valores são admissíveis, por exemplo grandes valores de $m_{1}$ e $m_{2}$ com pequenos valores de $h_{1}$ e $h_{2}$, que teriam alto custo), o usuário pode utilizar o programa em Excel desenvolvido pelos autores (disponível, bastando contactar o primeiro autor) para avaliar os desempenhos proporcionados para o gráfico pelas diversas combinações admissíveis e escolher melhor delas. Veja a Figura 2, que ilustra a interface com o usuário (primeira planilha do programa). As células cujos valores são dados de entrada estão em cinza escuro. A planilha calcula e fornece $\bar{h}, \bar{m}, \bar{m} / \bar{h}$, $T M A F$ e os perfis de TES em função de $\gamma$. A mera inspeção dos valores obtidos pode ser suficiente para a decisão. Como auxílio à decisão, são ainda fornecidos, sob as curvas de $T E S$, para cada valor de $\gamma$ : os fatores de redução no TES (razão $T E S_{\text {adapt }} / T E S_{F p}$ ); a eficiência relativa, razão entre as funções $g$ definidas em (6); e o ganho percentual de eficiência, calculado como $100 \times($ eficiência relativa -1$)$; tudo isso em relação ao gráfico $F p$ de referência. O usuário deve introduzir os valores dos limites de controle e de advertência, para estabelecer os melhores valores por tentativa e erro: experimentando valores e observando os resultados fornecidos pela planilha. Para evitar dúvidas sobre a localização do número de não-conformidades de cada amostra nas regiões do gráfico, é recomendável utilizar sempre limites com valores não inteiros, como feito na Tabela 2. Sugere-se começar com os valores de $L S C_{1}$ e $L S C_{2}$ (a planilha fornece valores iniciais para a busca: um valor mínimo para $L S C_{1}$ e um valor máximo para $L S C_{2}$ ), e depois experimentar valores para $L S A_{1}$ e $L S A_{2}$, observando o seu efeito nos valores de $\bar{m} / \bar{h}, T M A F$ e perfis de TES. Essa busca não é muito longa, pois os resultados são muito sensíveis aos valores dos limites, de modo que não há muitas opções de valores alternativos a serem testados: o usuário logo perceberá quando alterações de um desses limites começam a produzir valores muito grandes de $\bar{m} / \bar{h}$ ou TMAF, ou valores muito baixos de TES. Consegue-se assim um bom projeto rapidamente. Como a planilha de interface só exibe um projeto de cada vez, e o usuário pode querer armazenar resultados de projetos intermediários satisfatórios nesse processo de busca do melhor projeto, a interface possui ainda dois botões no canto superior direito, "Guardar resultado Adapt." e "Guardar resultado Fp". Clicando o mouse nesses botões, os parâmetros e os resultados do projeto corrente são armazenados em duas outras planilhas da mesma pasta, para facilidade de comparação posterior (as Tabelas 4 e 6 a seguir foram produzidas dessa forma). 


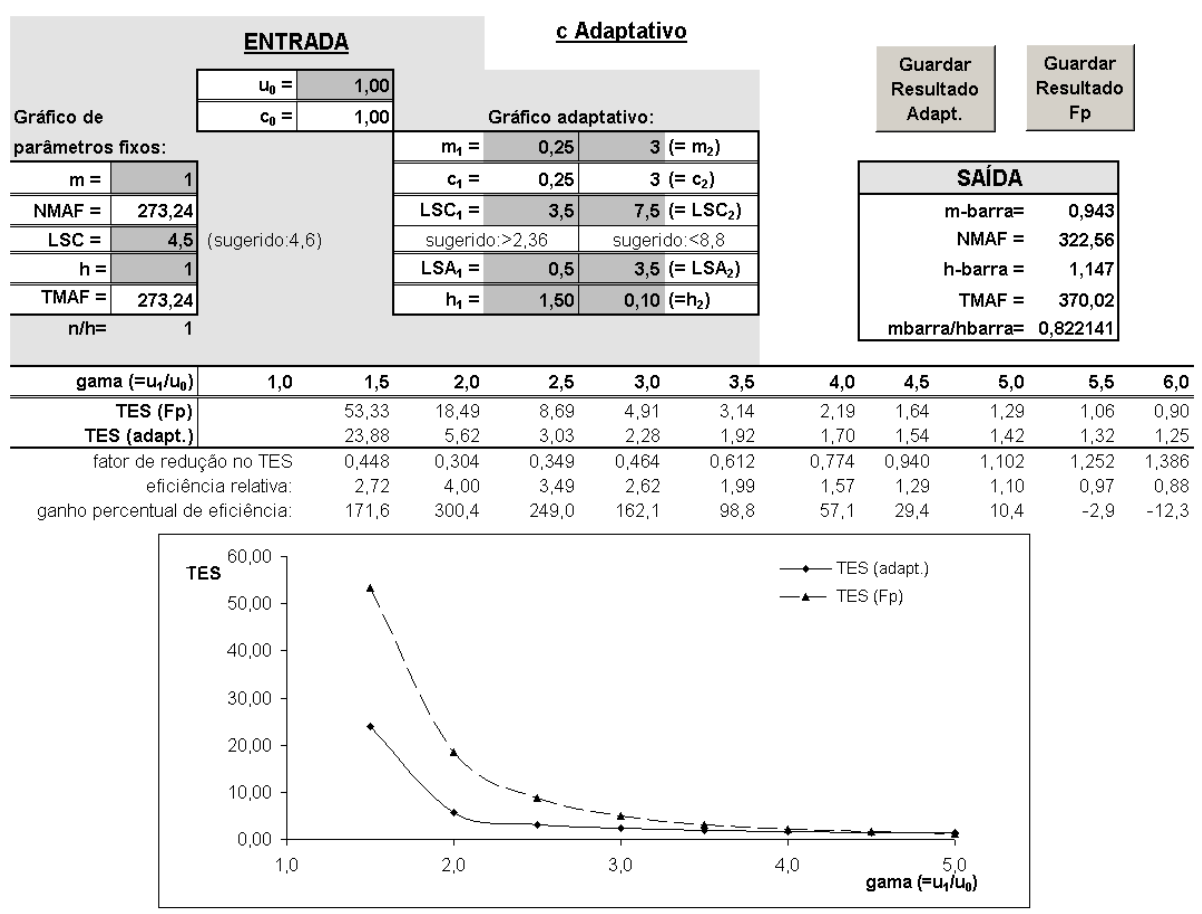

Figura 2 - Interface do programa de cálculo para obtenção dos projetos dos esquemas.

Para ilustrar o uso da planilha na obtenção de um bom projeto que satisfaça a um conjunto de restrições de ordem prática, veja-se o seguinte exemplo. Suponha um processo de pintura de carrocerias, em que o número médio de defeitos por carroceria (com o processo em controle) seja de 0,8 defeitos. Consideram-se os seguintes valores possíveis para o número de carrocerias em uma amostra: $\{1,2,4\}$, e os seguintes intervalos de tempo entre amostras: $\{2 \mathrm{~h}, 4 \mathrm{~h}, 8 \mathrm{~h}\}$. Porém, nem todas as combinações desses valores são possíveis: há uma restrição de examinar apenas, em média, 1 carroceria a cada 2 horas; ou seja, $\mathrm{m} / \mathrm{h}$ (e $\bar{m} / \bar{h})=0,5$.

Os gráficos de $c$ de parâmetros fixos possíveis com esses valores de $m$ e $h$ estão na Tabela 4, que apresenta, além dos valores de $m, h$ e $L S C$, o TMAF e os valores de TES quando $1,5 u_{0} \leq u_{1} \leq 4,0 u_{0}$. Esses valores são fornecidos pela planilha. Para cada gráfico, o $L S C$ escolhido foi o menor valor que satisfaz $T M A F \geq 200$. Cada projeto é identificado por uma letra, na primeira coluna, "Id.", da Tabela 4.

Tabela 4 - Projetos $F p$ possíveis no exemplo.

\begin{tabular}{|c|c|c|c|c|c|c|c|c|c|c|}
\hline \multirow{2}{*}{$I d$. } & \multirow{2}{*}{$m$} & \multirow{2}{*}{$h$} & \multirow{2}{*}{$L S C$} & \multirow{2}{*}{$m / h$} & \multirow{2}{*}{$T M A F$} & \multicolumn{5}{|c|}{ TES para rigual a } \\
\hline & & & & & & 1,5 & 2 & 2,5 & 3 & 4 \\
\hline$x$ & 1 & 2 & 4,5 & 0,500 & 1417,1 & 257,20 & 83,45 & 36,98 & 19,86 & 8,12 \\
\hline$y$ & 2 & 4 & 5,5 & 0,500 & 662,2 & 110,13 & 35,95 & 16,62 & 9,46 & 4,49 \\
\hline$z$ & 4 & 8 & 7,5 & 0,500 & 475,4 & 66,59 & 21,59 & 10,62 & 6,79 & 4,51 \\
\hline
\end{tabular}


É imediato ver que o projeto $z$ fornece TES's muito inferiores aos demais - o que é razoável, dado que tem o menor $T M A F$ dos três. Como trata-se de um valor satisfatório $(T M A F>200)$, o projeto $z$ é o preferível, dados seus menores TES's. Suponha porém que o usuário considere estes TES's ainda muito altos; nesse caso, os esquemas adaptativos surgem como uma alternativa. As combinações possíveis dos tamanhos de amostra e intervalos de amostragem admissíveis estão na Tabela 5. Note que, como $\left(m_{1} / h_{1}\right)<\bar{m} / \bar{h}<\left(m_{2} / h_{2}\right)$, não estão em consideração combinações em que $\left(m_{1} / h_{1}\right) \geq 0,5$ ou em que $\left(m_{2} / h_{2}\right) \leq 0,5$.

Tabela 5 - Combinações de tamanhos de amostra e intervalos de amostragem admissíveis na prática, no exemplo.

\begin{tabular}{ccccc}
\hline$I d$. & $m_{1}$ & $m_{2}$ & $h_{1}$ & $h_{2}$ \\
\hline$a$ & 1 & 2 & 8 & 2 \\
$b$ & 1 & 2 & 4 & 2 \\
$c$ & 1 & 4 & 8 & 4 \\
$d$ & 1 & 4 & 8 & 2 \\
$e$ & 1 & 4 & 4 & 2 \\
$f$ & 2 & 4 & 8 & 4 \\
$g$ & 2 & 4 & 8 & 2 \\
\hline
\end{tabular}

As combinações possíveis com $m_{1}=m_{2}$ (esquemas $V S I L$ e $V S I$ ) e com $h_{1}=h_{2}$ (esquema VSS) não são incluídas aqui, porém foram testadas e mostraram desempenho muito inferior ao das combinações $V p$ da Tabela 5.

Introduzindo cada uma das combinações na planilha e, para cada uma delas, buscando por tentativa e erro os melhores valores para os limites de controle e de advertência, o usuário obteve os projetos na Tabela 6 (os melhores que ele obteve para cada combinação).

Tabela 6 - Projetos $V p$ obtidos pelo usuário no exemplo.

\begin{tabular}{ccccccccccccccccc}
\hline \multirow{2}{*}{ Id. } & $m_{1}$ & $m_{2}$ & $h_{1}$ & $h_{2}$ & $L S C_{1}$ & $L A_{1}$ & $L S C_{2}$ & $L A_{2}$ & $\bar{m} / \bar{h}$ & $T M A F$ & \multicolumn{5}{c}{ TES para rigual $a$} \\
\hline$a$ & 1 & 2 & 8 & 2 & 4,5 & 0,5 & 4,5 & 0,5 & 0,473 & 207,7 & 35,25 & 13,95 & 8,38 & 6,19 & 4,49 \\
$b$ & 1 & 2 & 4 & 2 & 3,5 & 0,5 & 5,5 & 1,5 & 0,506 & 395,9 & 74,19 & 23,49 & 10,78 & 6,39 & 3,49 \\
$c$ & 1 & 4 & 8 & 4 & 5,5 & 0,5 & 7,5 & 2,5 & 0,489 & 567,3 & 53,02 & 16,89 & 9,69 & 7,29 & 5,69 \\
$d$ & 1 & 4 & 8 & 2 & 3,5 & 0,5 & 7,5 & 2,5 & 0,615 & 329,4 & 34,37 & 11,39 & 7,02 & 5,53 & 4,42 \\
$e$ & 1 & 4 & 4 & 2 & 5,5 & 1,5 & 7,5 & 3,5 & 0,485 & 854,2 & 56,09 & 14,87 & 8,03 & 5,82 & 4,21 \\
$f$ & 2 & 4 & 8 & 4 & 5,5 & 0,5 & 7,5 & 3,5 & 0,546 & 472,2 & 53,62 & 16,14 & 8,50 & 6,01 & 4,31 \\
$g$ & 2 & 4 & 8 & 2 & 5,5 & 0,5 & 7,5 & 3,5 & 0,680 & 379,0 & 39,03 & 11,45 & 6,40 & 4,90 & 3,92 \\
\hline
\end{tabular}

Os projetos $b, c$ e $f$ são bastante inferiores aos demais (possuem TES's maiores, de maneira geral) e podem ser descartados; dos restantes, com $\bar{m} / \bar{h}$ aproximadamente igual a 0,5 há 
apenas os projetos $a$ e $e$, sendo o último ligeiramente melhor para $\gamma \geq 2,5$ e o primeiro, melhor para $\gamma \leq 2,0$ (em particular, muito melhor para $\gamma=1,5$ ). A escolha entre eles dependerá da magnitude $\gamma^{*}$ de perturbação do processo que se considera importante detectar com rapidez. Porém há ainda outra possibilidade: os projetos $d$ e $g$ possuem os menores $T E S$ 's de todos, com valores de $\bar{m} / \bar{h}$ um pouco maiores. Examinando os TES's dos diversos projetos para a perturbação $\gamma^{*}$, o usuário contraporá "rapidez" e custo $(\bar{m} / \bar{h})$, para decidir.

A interpretação dos resultados da Tabela 6 pode ainda ser beneficiada através da medida $g=T E S \times(\bar{m} / \bar{h})$ proposta na seção 5 (equação (6)), que quantifica essa relação entre rapidez e custo. Veja a Tabela 7. O menor valor de $g$ para cada valor de $\gamma$ está realçado em negrito, indicando o projeto mais eficiente na detecção do aumento correspondente na freqüência média de não-conformidades. Essa tabela mostra que os projetos $d$ e $g$, a despeito de seus menores TES's, são menos eficientes que os projetos $a$ e $e$ : o aumento que eles demandam no custo $\bar{m} / \bar{h}$ (em relação aos projetos $a$ e $e$ ) é proporcionalmente maior que a redução que eles propiciam nos TES's. Outra maneira de ver isso é a seguinte: se no projeto $a$ (ou no projeto $e$ ) o usuário pudesse reduzir $h_{1}$ e $h_{2}$ numa mesma proporção até o ponto em que o valor de $\bar{m} / \bar{h}$, aumentando, se igualasse ao valor de $\bar{m} / \bar{h}$ do projeto $d$ (ou do projeto $g$ ), ele conseguiria com isso TES's menores que os do projeto $d$ (ou do projeto $g$ ). Claro que, como não há essa flexibilidade nos valores de $h_{1} \mathrm{e} h_{2}$, ele poderá ainda preferir um projeto menos eficiente, mas com menor TES, como $d$ ou $g$. A decisão final permanece uma questão de preferência do usuário.

Tabela 7 - Eficiência dos projetos $V p$ obtidos pelo usuário no exemplo.

\begin{tabular}{cccccc}
\hline \multirow{2}{*}{ Id. } & \multicolumn{5}{c}{ função g para jigual $a$} \\
\cline { 2 - 6 } & 1,5 & 2 & 2,5 & 3 & 4 \\
\hline$a$ & $\mathbf{1 6 , 6 7}$ & $\mathbf{6 , 6 0}$ & 3,96 & 2,93 & 2,12 \\
$b$ & 37,54 & 11,89 & 5,45 & 3,23 & $\mathbf{1 , 7 7}$ \\
$c$ & 25,93 & 8,26 & 4,74 & 3,56 & 2,78 \\
$d$ & 21,14 & 7,00 & 4,32 & 3,40 & 2,72 \\
$e$ & 27,20 & 7,21 & $\mathbf{3 , 8 9}$ & $\mathbf{2 , 8 2}$ & 2,04 \\
$f$ & 29,28 & 8,81 & 4,64 & 3,28 & 2,35 \\
$g$ & 26,54 & 7,79 & 4,35 & 3,33 & 2,67 \\
\hline
\end{tabular}

De qualquer forma, com qualquer um desses quatro melhores projetos $(a, d, e$ e $g)$, a redução dos TES's em relação ao melhor projeto convencional (gráfico de parâmetros fixos), que é o projeto $z$ da Tabela 4, é bastante significativa.

Mesmo assim, o ganho de eficiência proporcionado pelos projetos adaptativos neste exemplo foi menor que os ganhos dos projetos indicados na Tabela 2 (e verificáveis na Tabela 3), na comparação de desempenho entre esquemas (os projetos da Tabela 2 proporcionavam TES's duas a cinco vezes menores que os dos gráficos Fp de referência; os projetos adaptativos da Tabela 6 acima proporcionam reduções muito menos significativas). Uma das razões é o número limitado de alternativas para os parâmetros do gráfico, que leva naturalmente a 
soluções sub-ótimas (ou, de outra maneira, trata-se de soluções ótimas num conjunto factível mais restrito).

Por outro lado, como $\bar{m}<m_{2}$ e $\bar{h}>h_{2}$, pode ser razoável admitir valores para $m_{2}$ maiores que o maior valor de $m$ admissível para o gráfico $F p$ e, da mesma forma, valores para $h_{2}$ menores que o menor valor admissível para $m$, sem que isso implique necessariamente em valores de $\bar{m} / \bar{h}$ maiores que os valores de $m / h$ admissíveis para os gráficos $F p$. Restringir os tamanhos de amostra e tempos entre amostras para os gráficos adaptativos ao mesmo conjunto de valores que para os gráficos $F p$, como foi feito no exemplo, pode limitar demasiadamente as opções, eliminando boas alternativas, e colocando, assim, o esquema adaptativo em desvantagem na comparação.

Para buscar melhores projetos, então, poder-se-ia considerar, no caso do exemplo, $m_{2}=8$ e/ou $h_{2}=1$, o que fornece as combinações adicionais de $m_{1}, m_{2}, h_{1}$ e $h_{2}$ constantes da Tabela 8 .

Tabela 8 - Combinações adicionais de tamanhos de amostra e intervalos de amostragem, no exemplo.

\begin{tabular}{cccc}
\hline$m_{1}$ & $m_{2}$ & $h_{1}$ & $h_{2}$ \\
\hline 1 & 8 & 8 & 4 \\
1 & 8 & 4 & 2 \\
2 & 8 & 8 & 4 \\
2 & 8 & 8 & 2 \\
1 & 2 & 8 & 1 \\
1 & 2 & 4 & 1 \\
1 & 4 & 4 & 1 \\
2 & 4 & 8 & 1 \\
\hline
\end{tabular}

Com estas combinações adicionais, conseguem-se vários projetos melhores que os da Tabela 6. Para não alongar demasiadamente o exemplo, apenas o projeto mais eficiente de todos será descrito: veja a Tabela 9 .

Tabela 9 - Projeto $V p$ mais eficiente obtido pelo usuário no exemplo.

\begin{tabular}{|c|c|c|c|c|c|c|c|c|c|c|c|c|c|c|c|}
\hline \multirow{2}{*}{ Id. } & \multirow{2}{*}{$m_{l}$} & \multirow{2}{*}{$m_{2}$} & \multirow{2}{*}{$h_{1}$} & \multirow{2}{*}{$h_{2}$} & \multirow{2}{*}{$L S C_{l}$} & \multirow{2}{*}{$L A_{I}$} & \multirow{2}{*}{$L S C_{2}$} & \multirow{2}{*}{$L A_{2}$} & \multirow{2}{*}{$\bar{m} / \bar{h}$} & \multirow{2}{*}{$T M A F$} & \multicolumn{5}{|c|}{ TES para $\gamma$ igual a } \\
\hline & & & & & & & & & & & 1,5 & 2 & 2,5 & 3 & 4 \\
\hline \multirow[t]{2}{*}{$h$} & 1 & 4 & 4 & 1 & 3,5 & 1,5 & 6,5 & 3,5 & 0,503 & 195,2 & 26,21 & 9,50 & 5,81 & 4,39 & 3,17 \\
\hline & & & & & & & & & func & ão $g$ : & 13,18 & 4,78 & 2,92 & 2,21 & 1,59 \\
\hline
\end{tabular}

Este projeto fornece $T E S$ 's sensivelmente menores que todos os projetos da Tabela 6 , ao preço de um $T M A F$ menor (inclusive violando ligeiramente a restrição inicial $T M A F \geq 200$ ). Seu custo de amostragem médio, porém, é praticamente igual ao valor estabelecido pelo usuário $(\bar{m} / \bar{h}=0,5)$. Este é um exemplo da situação em que uma pequena violação de uma das restrições pode ser compensada por uma redução substancial no TES. Considerando 
apenas a medida $g=T E S \times(\bar{m} / \bar{h})$, este é o projeto mais eficiente que o usuário teria encontrado, para todos os valores de $\gamma$. A redução dos TES's em relação ao melhor projeto $F p$ (projeto $z$ da Tabela 4), vai de $20 \%$ (no caso de aumentos de $300 \%$ em $u_{0}$ ) até $60 \%$ (no caso de aumentos de $50 \%$ em $u_{0}$ ).

\section{Gráficos Adaptativos de $n p, p$ e $u$}

Considerando as mesmas suposições feitas no estudo das propriedades do gráfico de $c$, podem-se obter as seguintes expressões para as probabilidades de transição associadas ao gráfico adaptativo de $n p$ :

$$
\begin{array}{ll}
p_{i 1}^{(f)}=\sum_{j=0}^{\operatorname{int}\left(L S A_{i}\right)}\left(\begin{array}{c}
n_{i} \\
j
\end{array}\right) p_{f}^{j}\left(1-p_{f}\right)^{n_{i}-j} & i=1,2 ; f=0,1 \\
p_{i 2}^{(f)}=\sum_{j=\operatorname{int}\left(L S A_{i}\right)+1}^{\operatorname{int}\left(L S C_{i}\right)}\left(\begin{array}{c}
n_{i} \\
j
\end{array}\right) p_{f}^{j}\left(1-p_{f}\right)^{n_{i}-j} & i=1,2 ; f=0,1
\end{array}
$$

onde $p_{f}$ é a fração média de defeituosos produzida pelo processo, o índice $f$ indicando o estado do processo. Assim, durante a fase em controle, $p_{f}=p_{0} \mathrm{e}$, durante a fase fora de controle, $p_{f}=p_{1}\left(p_{1}>p_{0}\right)$. Além disso, $n, n_{1}$ e $n_{2}$ são, respectivamente, o tamanho fixo de amostra do gráfico de $n p$ e o menor e maior tamanhos de amostra do gráfico adaptativo de $n p$.

Com exceção das expressões das probabilidades de transição, o modelo matemático para obtenção das medidas de desempenho, o projeto e a operação do gráfico adaptativo de $n p$ são idênticos aos do gráfico adaptativo de $c$. Além disso, devido à aproximação entre as distribuições binomial e de Poisson, para valores pequenos de $p_{0}$, as probabilidades de transição em (7) e (8) tornam-se praticamente iguais aos valores fornecidos pelas expressões (1) e (2) com $u_{f}=n_{i} p_{f} / m_{i}, f=1,2$. Conseqüentemente, os TES's e os TMAF's dos gráficos adaptativos e não-adaptativos de $n p$ são quase iguais aos $T E S$ 's e os $T M A F$ 's dos gráficos de $c$ correspondentes.

Portanto, para qualquer par de valores $\left(n, p_{0}\right)$ tal que o produto $n p_{0}$ coincida com algum dos valores de $c_{0}$ na Tabela 2 , e desde que $p_{0}$ seja suficientemente pequeno $\left(p_{0} \leq 0,0200\right)$, podem-se obter projetos $F p$ e adaptativos de gráficos de $n p$, correspondentes aos projetos de gráficos de $c$ da Tabela 2, através das relações: $n_{i}=m_{i} u_{0} / p_{0}=m_{i} n$ para $i=1$, 2. Como no gráfico de $n p$ os tamanhos de amostra devem ser inteiros, os $n_{i}$ 's devem ser arredondados para o inteiro mais próximo, mas o efeito desse arredondamento no desempenho do gráfico será muito pequeno, dada a magnitude dos tamanhos de amostra envolvidos. Os demais parâmetros $\left(h_{i}, L S C_{i}\right.$ e $\left.L S A_{i}\right)$ permanecem os mesmos do gráfico adaptativo de $c$. Para o gráfico de controle $n p F p$, tem-se que $n=c_{0} / p_{0}$, sendo seu limite superior de controle igual ao do gráfico $c F p$.

As conclusões apresentadas na Seção 4, quando da comparação entre os esquemas de monitoramento para os gráficos de $c$, também são válidas para os gráficos de $n p$, quando a fração defeituosa $p_{0}$ é pequena; os autores repetiram a comparação entre os esquemas para gráficos de $n p$, para $p_{0}=0,0025$ e $p_{0}=0,0200$, e os resultados encontrados foram aproximadamente iguais aos resultados para os gráficos de $c \operatorname{com} m u_{0}=n p_{0}$. 
A obtenção de um projeto para o gráfico adaptativo de $n p$ na prática pode seguir o mesmo procedimento apresentado na Seção 5 para o projeto do gráfico de $c$. A mesma planilha pode ser utilizada, bastando substituir as expressões das probabilidades de transição $p_{11}, p_{12}, p_{21} \mathrm{e}$ $p_{22}$ pelas expressões (26) e (27) acima. No caso do gráfico de $n p$, o número de alternativas para os tamanhos de amostra é geralmente maior que no caso do gráfico de $c$, o que torna a busca mais longa; essa questão é administrável resolvendo o problema em etapas: primeiro admitindo apenas grandes variações no tamanho de amostra (por exemplo, de $100 \mathrm{em} 100$ unidades), depois experimentando variações menores em torno da primeira solução. Em compensação, esse maior número de alternativas pode levar a melhores soluções (com resultados mais próximos aos da solução ótima).

Os gráficos adaptativos de $u$ e de $p$ podem ser obtidos a partir dos gráficos adaptativos de $c$ e de $n p$, simplesmente dividindo $x_{k}, L S C_{i}$ e $L S A_{i}$ pelo tamanho de amostra $m_{i}$ ou $n_{i}$. Cabe ao usuário decidir se considera esta complexidade adicional compensada pela vantagem de ter um único valor para a linha média, e estatísticas diretamente comparáveis independentemente do tamanho de amostra.

\section{Conclusões}

Os gráficos adaptativos por atributos são mais rápidos que os gráficos tradicionais de parâmetros fixos na detecção de deteriorações de diferentes magnitudes na qualidade do processo, sem requererem maior volume de inspeção ou oferecerem menor proteção contra alarmes falsos. Como conseqüência, para alcançar a mesma rapidez de deteç̧ão proporcionada pelos gráficos adaptativos, os gráficos de parâmetros fixos requerem maior volume de inspeção por unidade de tempo e/ou oferecem menos proteção contra alarmes falsos. Assim, fixando-se uma freqüência de alarmes falsos, o ganho obtido com o esquema adaptativo pode ser explorado em duas direções: conseguir detecção mais rápida de descontroles sem aumentar o custo de amostragem, ou reduzir o custo de amostragem sem reduzir a rapidez de detecção de descontroles.

A magnitude desse ganho depende do projeto (conjunto de valores para os parâmetros) do gráfico adaptativo. O procedimento proposto na Seção 5 permite ao usuário obter o melhor projeto para um leque de valores operacionalmente viáveis do tamanho de amostra e do intervalo de amostragem. Esse procedimento se torna simples e rápido caso o usuário disponha de uma planilha em Excel que calcule e apresente as diversas medidas de desempenho para cada conjunto de valores dos parâmetros de projeto. Essa planilha está disponível, bastando contactar o primeiro autor.

Este artigo tratou do projeto dos gráficos adaptativos de $c$, de $n p$, de $u$ e de $p$, com um, dois ou todos os parâmetros variáveis. Isto contempla todos os gráficos usuais por atributos, e as situações práticas em que não seja possível variar algum (ou alguns) dos parâmetros.

As vantagens do esquema adaptativo se tornam tão mais relevantes quanto maior for o nível de exigência sobre o processo e quanto mais importante for, em decorrência, detectar alterações de menor magnitude no seu nível de qualidade. No caso de controle por atributos, essa relevância pode ser ainda maior que no caso de controle por variáveis, devido aos grandes tamanhos de amostra tipicamente requeridos. 


\section{Agradecimentos}

Somos gratos a dois revisores anônimos cuja revisão cuidadosa levou à eliminação de várias imperfeições deste artigo. Esta pesquisa teve o apoio financeiro do CNPq e da CAPES.

\section{Referências Bibliográficas}

(1) Bissel, A.F. (1988). Control chart limits for attributes and events. Journal of Applied Statistics, 15(1), 97-105.

(2) Costa, A.F.B. (1994). $\bar{X}$ Charts with Variable Sample Size. Journal of Quality Technology, 26, 155-163.

(3) Costa, A.F.B. (1997). $\bar{X}$ Charts with Variable Sample Size and Sampling Intervals. Journal of Quality Technology, 29, 197-204.

(4) Costa, A.F.B. (1998a). Gráficos de Controle $\bar{X}$ para Processos Robustos. Gestão\&Produção, 5(3), 259-271.

(5) Costa, A.F.B. (1998b). Joint $\bar{X}$ and $R$ Charts with Variable Parameters. IIE Transactions, 30, 505-514.

(6) Costa, A.F.B. (1999a). $\bar{X}$ Charts with Variable Parameters. Journal of Quality Technology, 31, 408-41.

(7) Costa, A.F.B. (1999b). Joint $\bar{X}$ and $R$ Charts with Variable Sample Size and Sampling Intervals. Journal of Quality Technology, 31, 387-397.

(8) Costa, A.F.B.; Epprecht, E.K. \& Carpinetti, L.C.R. (2004). Controle Estatístico de Qualidade. Atlas, São Paulo, SP.

(9) De Magalhães, M.S.; Costa, A.F.B. \& Epprecht, E.K. (2002). Constrained Optimization Model for the Design of an Adaptive $\bar{X}$ Chart. International Journal of Production Research, 40(13), 3199-3218.

(10) De Magalhães, M.S.; Epprecht, E.K. \& Costa, A.F.B. (2001). Economic Design of a $\mathrm{V}_{\mathrm{p}}$ $\bar{X}$ Chart. International Journal of Production Economics, 74, 191-200.

(11) De Magalhães, M.S. \& Moura Neto, F.D. (2004). Joint Economic Model for Totally Adaptive X-bar and R charts (no prelo). European Journal of Operational Research.

(12) Epprecht, E.K. \& Costa, A.F.B. (2001). Adaptive Sample Size Control Charts for Attributes. Quality Engineering, 13(3), 465-473.

(13) Epprecht, E.K.; Costa, A.F.B. \& Mendes, F.C.T. (2003). Adaptive Control Charts for Attributes. IIE Transactions, 35(6), 567-582.

(14) Epprecht, E.K. \& Santos, A.B. (1998). Um Método Simples para o Projeto Ótimo de Gráficos de $\bar{X}$. Gestão\&Produção, 5(3), 206-220.

(15) Montgomery, D.C. (2001). Introduction to Statistical Quality Control. $4^{\text {a }}$ edição. John Wiley \& Sons, Inc., New York, NY. 
(16) Porteus, E.L. \& Angelus, A. (1997). Opportunities for Improved Statistical Process Control. Management Science, 43, 1214-1228.

(17) Prabhu, S.S.; Runger, G.C. \& Keats, J.B. (1993). An Adaptive Sample Size $\bar{X}$ Chart. International Journal of Production Research, 31, 2895-2909.

(18) Prabhu, S.S.; Montgomery, D.C. \& Runger, G.C. (1994). A Combined Adaptive Sample Size and Sampling Interval $\bar{X}$ Control Scheme. Journal of Quality Technology, 26, 164-176.

(19) Rendtel, U. (1990). Variable Sampling Intervals and Sample Sizes. Statistical Papers, 31, 103-118.

(20) Reynolds, M.R., Jr. (1989). Optimal Variable Sampling Interval Control Charts. Sequential Analysis, 8, 361-379.

(21) Reynolds, M.R., Jr. (1995). Evaluating Properties of Variable Sampling Interval Control Charts. Sequential Analysis, 14, 59-97.

(22) Reynolds, M.R., Jr.; Amin, R.W.; Arnold, J.C. \& Nachlas, J.A. (1988). $\bar{X}$ Charts with Variable Sampling Intervals. Technometrics, 30, 181-192.

(23) Reynolds, M.R., Jr.; Amin, R.W. \& Arnold, J.C. (1990). CUSUM Charts with Variable Sampling Intervals. Technometrics, 32, 371-384.

(24) Runger, G.C. \& Pignatiello, J.J., Jr. (1991). Adaptive Sampling for Process Control. Journal of Quality Technology, 23, 135-155.

(25) Saccucci, M.S.; Amin, R.W. \& Lucas, J.M. (1992). Exponentially Weighted Moving Average Control Schemes with Variable Sampling Intervals. Communications in Statistics - Simulation and Computation, 21, 627-657.

(26) Tagaras, G. (1998). A Survey of Recent Developments in the Design of Adaptive Control Charts. Journal of Quality Technology, 30, 212-231.

(27) Vaughan, T.S. (1993). Variable Sampling Interval $n p$ Process Control Chart. Communications in Statistics - Theory and Methods, 22, 147-167.

(28) Zimmer, L.S.; Montgomery, D.C. \& Runger, G.C. (1998). Three-State Sample Size $\bar{X}$ Chart. International Journal of Production Research, 36, 733-743. 